\title{
Awareness of Zoonotic Diseases among Livestock Farmers in Budgam District of Kashmir Valley, India
}

\author{
Insha Mir* and Abdul Hai Bhat \\ Department of Veterinary \& Animal Husbandry Extension Education, \\ Sher-e-Kashmir University of Agricultural Sciences andTechnology of Kashmir, India \\ *Corresponding author
}

\section{A B S T R A C T}

Keywords

Awareness, livestock farmers, zoonotic diseases

Article Info

Accepted:

05 April 2020

Available Online:

10 May 2020
The study was conducted to assess the awareness of zoonotic diseases among livestock farmers in Budgam district of Kashmir valley.320 respondents were selected randomly and interviewed with a pretested questionnaire, which contained both open and close ended questions on different aspects of zoonotic diseases regarding awareness. Results regarding transmission of rabies through dog bite (16.56\%), need of post - exposure vaccination (9.69\%) were aware. Also (53.44\%) livestock farmers were aware of the occurrence of abortion due to brucellosis and availability of brucellosis vaccine $(68.44 \%)$ has an access in their area. Only $(0.94 \%)$ had suffered from zoonotic diseases in past. Safety measures were rarely practiced by livestock farmers. Overall awareness level was found low among livestock farmers.

\section{Introduction}

Zoonoses are defined as those diseases that are naturally transmitted between people and vertebrate animals which constitute a diverse group of viral, bacterial, ricketssial, fungal, parasitic, and prion disease with a variety of animal reservoirs, including wild life, livestock, pet animals, and birds (Nkuchia et al., 2007). The transmission may occur through direct contact with the animal, through vectors such as mosquitoes, fleas or ticks or through food or water contamination and had both direct and indirect effects on livestock health and its production (Smiths and Cutler, 2004). Zoonotic diseases cause morbidity and mortality in people, and is also imposing significantly losses to the livestock sector that account about $75 \%$ all emerging pathogens.

Many zoonotic diseases that are overlooked in endemic settings are not universally neglected. With an example of 
Brucellosis,well-studied and eradicated from several countries within highly developed commercial livestock sectors (Dean et al., 2012). Endemic zoonoses remain widely neglected in many low-income settings because their impact is borne largely by impoverished and marginalized communities (Molyneux et al., 2011). They disproportionately affect people who are not only at high risk of pathogen exposure but also have little access to adequate primary healthcare (ILRI, 2012).

Certain occupations have a higher risk of zoonoses (Schelling et al., 2003; Swai and Schoonman, 2009) like occupational zoonoses that result in exposure of humans to animal diseases during work. An estimated 320,000 occupationally related deaths from infectious diseases are reported yearly worldwide (Haagsma et al., 2011). There is likelihood that majority of the animals brought for slaughter increase the risk of infection to abattoir workers (Swai et al., 2010).

Within the human activities like working with animals in their sheds, improper disposal of waste from animal sheds, skinning of infected animals, disposal of infected material from the diseased animals and poor personnel hygiene practices have been reported to be an important risk factors contributing to frequent outbreaks of zoonotic diseases in humans. Even though most of the zoonotic diseases that remain undiagnosed and result into enormous suffering and death of thousands of children and adults annually.

In most of the under developed and developing countries farming practices, low education level, culture and eating habits, presence of reservoir population, inadequate disease control programmes and lack of knowledge about disease burden have been mostly reported to be associated with persistence of zoonotic diseases (Asbjer,
2009). Lack of awareness with regard to zoonotic diseases is one of the most important reasons for the frequent outbreaks of zoonotic diseases in people in general.

\section{Materials and Methods}

Budgam district was purposefully selected for the study. A list of 8 blocks chosen for the study was randomly selected. From the selected 8 blocks, randomly 2 villages were selected from each block. Thus, a total 16 villages were randomly selected in all.

From each randomly selected village, 20 respondents were taken into confidence and total respondents were 320 . The data was tabulated and analyzed using statistical package for the Social Science (SPSS) version 20 developed by IBM Company, USA.

\section{Results and Discussion}

Rabies and Brucellosis are the two most common diseases of zoonotic importance and the awareness regarding these diseases was found by $(48.44 \%)$ and statistically found nonsignificant difference among respondents in different blocks. The awareness level of farmers regarding transmission of rabies was taken into consideration and was indicated that rabies transmission occurred by bite of rabid dog only $(16.56 \%)$ agree and by direct contact only (45.00\%) agree and maximum respondents in Narbal block were having awareness regarding this and by saliva $(82.19 \%)$ agree and in Khag and Beerwah block least awareness was found regarding this statement.

Regarding awareness about post wound treatment of rabies it was found $(33.75 \%)$ wash with plain water, $(61.56 \%)$ wash with dettol/savlon, (23.13\%) wash with soap but still $(10.63 \%)$ farmers opined to apply chili 
powder on it which is a mere misconception, and there is a need to educate the people on this aspect. Only (9.69\%) of the respondents were aware about the need of post-dog bite vaccination in humans.

Brucellosis is another common disease of animals which is zoonotic in nature and can cause economic loss as well as health hazard to the farmers. However, (53.44\%) livestock farmers were aware of the fact that animals may abort in the third trimester of their pregnancy due to brucellosis and showed a non-significant variation.

Now-a-days, prophylactic vaccine is available for female dairy animals as a preventive measure, and about (68.44\%) of respondents availed that vaccine against brucellosis is available in their area. When farmers were asked about the disease/s that they acquired from their animals, it was revealed that about $(0.94 \%)$ respondents were suffered in Beerwah, B.K Pora, Nagam blocks and it was a skin infection.

Apart from all, zoonotic diseases are also transmitted from vegetables/fruits as well and only $(7.19 \%)$ respondents were aware and people of Budgam block was well aware regarding this fact. And the fact of above mentioned diseases were in agreement with Ananthnarayan and Paniker (2013) and revealed that livestock farmers may get rabies and brucellosis from animals and clinically manifest as an acute or chronic form.

Veterinary seeking service regarding zoonotic diseases is important for availing information. Testing of herd against zoonotic diseases is important especially in outbreaks so respondents were investigated and was found that $(46.88 \%)$ tested their herd against zoonotic diseases and statistically it was found non-significant difference. As a preventive measure $(85.63 \%)$ of the respondents used to vaccinate their herd against zoonotic disease.

Also medical advice is essential for zoonotic diseases and the maximum respondents (11.88\%) were found in Chadoora and Khag block who seek medical advice when they are sick.

As a preventive measure farmers should have knowledge regarding safety rearing practices by using protective clothing/gloves/mask, proper boiling of milk before drinking, proper cooking of raw meat before eating, proper washing of hands after work with animals before eating and before food preparation, quarantine of animals, avoid working with animals when they are sick, weak, pregnant and old, avoid eating of dead and diseased meat products, avoid living with animals under the same roof in a house, keeping healthy animals away from infected animals, Fumigation of animal houses, avoid bathing of children in the same pond where animals are cleaned/bathed, proper disposal of diseased and dead animals by deep burying, Anti-rabies vaccination of farmers, cleaning and disinfection of diseased animal sheds, health hazards related to animal work and by investigation it was found that $(15.31 \%)$, $(87.19 \%), \quad(100 \%), \quad(73.75 \%), \quad(5.63 \%)$, $(36.88 \%), \quad(100 \%), \quad(93.75 \%), \quad(62.50 \%)$, (0.63\%), (35.31\%), (61.25\%), (78.13\%), $(97.19 \%)$ and $(3.44 \%)$ respectively. When compared with Adesiji et al., (2005) it was revealed that high level of contact with animals is most prevailing reason for spread of zoonotic diseases. Also Compared with Junaid et al., (2015) assessed that livestock farmers should wash their hands before and after dealing with animals, also personal hygiene plays an integral role, wearing of protective clothing can reduce the risk of zoonotic diseases. 
Table.1 Knowledge regarding awareness about Rabies and Brucellosis

\begin{tabular}{|c|c|c|c|c|c|c|c|c|c|}
\hline \multirow[t]{2}{*}{ Awareness about Rabies \& Brucellosis } & \multicolumn{8}{|c|}{ Blocks } & \multirow[t]{2}{*}{ Pooled } \\
\hline & Budgam & Beerwah & Chadoora & Khansahib & Khag & B K Pora & Narbal & Nagam & \\
\hline $\mathbf{n}$ & 40 & 40 & 40 & 40 & 40 & 40 & 40 & 40 & 320 \\
\hline Aware & $\begin{array}{c}13 \\
(32.50)\end{array}$ & $\begin{array}{c}19 \\
(47.50)\end{array}$ & $\begin{array}{c}23 \\
(57.50)\end{array}$ & $\begin{array}{c}23 \\
(57.50)\end{array}$ & $\begin{array}{c}19 \\
(47.50)\end{array}$ & $\begin{array}{c}17 \\
(42.50)\end{array}$ & $\begin{array}{c}23 \\
(57.50)\end{array}$ & $\begin{array}{c}18 \\
(45.00)\end{array}$ & $\begin{array}{c}155 \\
(48.44)\end{array}$ \\
\hline Unaware & $\begin{array}{c}27 \\
(67.50)\end{array}$ & $\begin{array}{c}21 \\
(52.50)\end{array}$ & $\begin{array}{c}17 \\
(42.50)\end{array}$ & $\begin{array}{c}17 \\
(42.50)\end{array}$ & $\begin{array}{c}21 \\
(52.50)\end{array}$ & $\begin{array}{c}23 \\
(57.50)\end{array}$ & $\begin{array}{c}17 \\
(42.50)\end{array}$ & $\begin{array}{c}22 \\
(55.00)\end{array}$ & $\begin{array}{c}165 \\
(51.56)\end{array}$ \\
\hline
\end{tabular}

(Figures in parenthesis indicate percentage, $\mathrm{n}$ indicate sample size $)(\mathrm{P}$ value $=0.267)$

Sources of rabies infection

Table.2 Bite of rabid dog

\begin{tabular}{|c|c|c|c|c|c|c|c|c|c|}
\hline \multirow[t]{2}{*}{ Rabies via Bite of rabid dog } & \multicolumn{8}{|c|}{ Blocks } & \multirow[t]{2}{*}{ Pooled } \\
\hline & Budgam & Beerwah & Chadoora & Khansahib & Khag & B K Pora & Narbal & Nagam & \\
\hline $\mathbf{n}$ & 40 & 40 & 40 & 40 & 40 & 40 & 40 & 40 & 320 \\
\hline Agree & $\begin{array}{c}4 \\
(10.00)\end{array}$ & $\begin{array}{c}7 \\
(17.50)\end{array}$ & $\begin{array}{c}2 \\
(5.00)\end{array}$ & $\begin{array}{c}5 \\
(12.50)\end{array}$ & $\begin{array}{c}6 \\
(15.00)\end{array}$ & $\begin{array}{c}8 \\
(20.00)\end{array}$ & $\begin{array}{c}11 \\
(27.50)\end{array}$ & $\begin{array}{c}10 \\
(25.00)\end{array}$ & $\begin{array}{c}53 \\
(16.56)\end{array}$ \\
\hline Disagree & $\begin{array}{c}1 \\
(2.50)\end{array}$ & $\begin{array}{c}1 \\
(2.50)\end{array}$ & $\begin{array}{c}2 \\
(5.00)\end{array}$ & $\begin{array}{c}3 \\
(7.50)\end{array}$ & $\begin{array}{c}1 \\
(2.50)\end{array}$ & $\begin{array}{c}5 \\
(12.50)\end{array}$ & $\begin{array}{c}17 \\
(42.50)\end{array}$ & $\begin{array}{c}6 \\
(15.00)\end{array}$ & $\begin{array}{c}36 \\
(11.25)\end{array}$ \\
\hline Don't know & $\begin{array}{c}35 \\
(87.50)\end{array}$ & $\begin{array}{c}32 \\
(80.00)\end{array}$ & $\begin{array}{c}36 \\
(90.00)\end{array}$ & $\begin{array}{c}32 \\
(80.00)\end{array}$ & $\begin{array}{c}33 \\
(82.50)\end{array}$ & $\begin{array}{c}27 \\
(67.50)\end{array}$ & $\begin{array}{c}12 \\
(30.00)\end{array}$ & $\begin{array}{c}24 \\
(60.00)\end{array}$ & $\begin{array}{c}231 \\
(72.19)\end{array}$ \\
\hline
\end{tabular}

(Figures in parenthesis indicate percentage, $\mathrm{n}$ indicate sample size $)(\mathrm{P}$ value $=0.001)$

Table.3 Rabies via contact with rabid dog

\begin{tabular}{|c|c|c|c|c|c|c|c|c|c|}
\hline \multirow[t]{2}{*}{ Rabies via Contact with rabid dog } & \multicolumn{8}{|c|}{ Blocks } & \multirow[t]{2}{*}{ Pooled } \\
\hline & Budgam & Beerwah & Chadoora & Khansahib & Khag & B K Pora & Narbal & Nagam & \\
\hline $\mathbf{n}$ & 40 & 40 & 40 & 40 & 40 & 40 & 40 & 40 & 320 \\
\hline Agree & $\begin{array}{c}24 \\
(60.00)\end{array}$ & $\begin{array}{c}16 \\
(40.00)\end{array}$ & $\begin{array}{c}17 \\
(42.50)\end{array}$ & $\begin{array}{c}15 \\
(37.50)\end{array}$ & $\begin{array}{c}18 \\
(45.00)\end{array}$ & $\begin{array}{c}16 \\
(40.00)\end{array}$ & $\begin{array}{c}22 \\
(55.00)\end{array}$ & $\begin{array}{c}16 \\
(40.00)\end{array}$ & $\begin{array}{c}144 \\
(45.00)\end{array}$ \\
\hline Disagree & $\begin{array}{c}4 \\
(10.00)\end{array}$ & $\begin{array}{c}9 \\
(22.50)\end{array}$ & $\begin{array}{c}13 \\
(32.50)\end{array}$ & $\begin{array}{c}18 \\
(45.00)\end{array}$ & $\begin{array}{c}17 \\
(42.50)\end{array}$ & $\begin{array}{c}16 \\
(40.00)\end{array}$ & $\begin{array}{c}11 \\
(27.50)\end{array}$ & $\begin{array}{c}18 \\
(45.00)\end{array}$ & $\begin{array}{c}106 \\
(33.13)\end{array}$ \\
\hline Don't know & $\begin{array}{c}12 \\
(30.00)\end{array}$ & $\begin{array}{c}15 \\
(37.50)\end{array}$ & $\begin{array}{c}10 \\
(25.00)\end{array}$ & $\begin{array}{c}7 \\
(17.50)\end{array}$ & $\begin{array}{c}5 \\
(12.50)\end{array}$ & $\begin{array}{c}8 \\
(20.00)\end{array}$ & $\begin{array}{c}7 \\
(17.50)\end{array}$ & $\begin{array}{c}6 \\
(15.00)\end{array}$ & $\begin{array}{c}70 \\
(21.88)\end{array}$ \\
\hline
\end{tabular}

(Figures in parenthesis indicate percentage, $\mathrm{n}$ indicate sample size $)(\mathrm{P}$ value $=0.023)$ 
Table.4 Saliva of rabid dog

\begin{tabular}{|c|c|c|c|c|c|c|c|c|c|}
\hline \multirow[t]{2}{*}{ Saliva of rabid dog } & \multicolumn{8}{|c|}{ Blocks } & \multirow[t]{2}{*}{ Pooled } \\
\hline & Budgam & Beerwah & Chadoora & Khansahib & Khag & B K Pora & Narbal & Nagam & \\
\hline $\mathbf{N}$ & 40 & 40 & 40 & 40 & 40 & 40 & 40 & 40 & 320 \\
\hline Agree & $\begin{array}{c}32 \\
(80.00)\end{array}$ & $\begin{array}{c}37 \\
(92.50)\end{array}$ & $\begin{array}{c}34 \\
(85.00)\end{array}$ & $\begin{array}{c}33 \\
(82.50)\end{array}$ & $\begin{array}{c}32 \\
(80.00)\end{array}$ & $\begin{array}{c}30 \\
(75.00)\end{array}$ & $\begin{array}{c}31 \\
(77.50)\end{array}$ & $\begin{array}{c}34 \\
(85.00)\end{array}$ & $\begin{array}{c}263 \\
(82.19)\end{array}$ \\
\hline Disagree & $\begin{array}{c}1 \\
(2.50)\end{array}$ & $\begin{array}{c}1 \\
(2.50)\end{array}$ & $\begin{array}{c}3 \\
(7.50)\end{array}$ & $\begin{array}{c}2 \\
(5.00)\end{array}$ & $\begin{array}{c}5 \\
(12.50)\end{array}$ & $\begin{array}{c}6 \\
(15.00)\end{array}$ & $\begin{array}{c}6 \\
(15.00)\end{array}$ & $\begin{array}{c}2 \\
(5.00)\end{array}$ & $\begin{array}{c}26 \\
(8.13)\end{array}$ \\
\hline Don't know & $\begin{array}{c}7 \\
(17.50)\end{array}$ & $\begin{array}{c}2 \\
(5.00)\end{array}$ & $\begin{array}{c}3 \\
(7.50)\end{array}$ & $\begin{array}{c}5 \\
(12.50)\end{array}$ & $\begin{array}{c}3 \\
(7.50)\end{array}$ & $\begin{array}{c}4 \\
(10.00)\end{array}$ & $\begin{array}{c}3 \\
(7.50)\end{array}$ & $\begin{array}{c}4 \\
(10.00)\end{array}$ & $\begin{array}{c}31 \\
(9.69)\end{array}$ \\
\hline
\end{tabular}

(Figures in parenthesis indicate percentage, $\mathrm{n}$ indicate sample size $)(\mathrm{P}$ value $=0.375)$

Awareness with regard to post wound treatment of a dog bite

Table.5 Wash with plain water

\begin{tabular}{|c|c|c|c|c|c|c|c|c|c|}
\hline \multirow[t]{2}{*}{ Wound Washing with plain water } & \multicolumn{8}{|c|}{ Blocks } & \multirow[t]{2}{*}{ Pooled } \\
\hline & Budgam & Beerwah & Chadoora & Khansahib & Khag & B K Pora & Narbal & Nagam & \\
\hline $\mathbf{N}$ & 40 & 40 & 40 & 40 & 40 & 40 & 40 & 40 & 320 \\
\hline No & $\begin{array}{c}20 \\
(50.00)\end{array}$ & $\begin{array}{c}23 \\
(57.50)\end{array}$ & $\begin{array}{c}26 \\
(65.00)\end{array}$ & $\begin{array}{c}26 \\
(65.00)\end{array}$ & $\begin{array}{c}26 \\
(65.00)\end{array}$ & $\begin{array}{c}28 \\
(70.00)\end{array}$ & $\begin{array}{c}29 \\
(72.50)\end{array}$ & $\begin{array}{c}34 \\
(85.00)\end{array}$ & $\begin{array}{c}212 \\
(66.25)\end{array}$ \\
\hline Yes & $\begin{array}{c}20 \\
(50.00)\end{array}$ & $\begin{array}{c}17 \\
(42.50)\end{array}$ & $\begin{array}{c}14 \\
(35.00)\end{array}$ & $\begin{array}{c}14 \\
(35.00)\end{array}$ & $\begin{array}{c}14 \\
(35.00)\end{array}$ & $\begin{array}{c}12 \\
(30.00)\end{array}$ & $\begin{array}{c}11 \\
(27.50)\end{array}$ & $\begin{array}{c}6 \\
(15.00)\end{array}$ & $\begin{array}{c}108 \\
(33.75)\end{array}$ \\
\hline
\end{tabular}

(Figures in parenthesis indicate percentage, $\mathrm{n}$ indicate sample size $)(\mathrm{P}$ value $=0.063)$

Table.6 Wash with soap

\begin{tabular}{|c|c|c|c|c|c|c|c|c|c|}
\hline \multirow[t]{2}{*}{ Wound Washing with soap } & \multicolumn{8}{|c|}{ Blocks } & \multirow[t]{2}{*}{ Pooled } \\
\hline & Budgam & Beerwah & Chadoora & Khansahib & Khag & B K Pora & Narbal & Nagam & \\
\hline $\mathbf{N}$ & 40 & 40 & 40 & 40 & 40 & 40 & 40 & 40 & 320 \\
\hline No & $\begin{array}{c}22 \\
(55.00)\end{array}$ & $\begin{array}{c}29 \\
(72.50)\end{array}$ & $\begin{array}{c}25 \\
(62.50)\end{array}$ & $\begin{array}{c}35 \\
(87.50)\end{array}$ & $\begin{array}{c}33 \\
(82.50)\end{array}$ & $\begin{array}{c}36 \\
(90.00)\end{array}$ & $\begin{array}{c}34 \\
(85.00)\end{array}$ & $\begin{array}{c}32 \\
(80.00)\end{array}$ & $\begin{array}{c}246 \\
(76.88)\end{array}$ \\
\hline Yes & $\begin{array}{c}18 \\
(45.00)\end{array}$ & $\begin{array}{c}11 \\
(27.50)\end{array}$ & $\begin{array}{c}15 \\
(37.50)\end{array}$ & $\begin{array}{c}5 \\
(12.50)\end{array}$ & $\begin{array}{c}7 \\
(17.50)\end{array}$ & $\begin{array}{c}4 \\
(10.00)\end{array}$ & $\begin{array}{c}6 \\
(15.00)\end{array}$ & $\begin{array}{c}8 \\
(20.00)\end{array}$ & $\begin{array}{c}74 \\
(23.13)\end{array}$ \\
\hline
\end{tabular}

(Figures in parenthesis indicate percentage, $\mathrm{n}$ indicate sample size $)(\mathrm{P}$ value $=0.001)$ 
Table.7 Wash with savlon/Dettol

\begin{tabular}{|c|c|c|c|c|c|c|c|c|c|}
\hline \multirow[t]{2}{*}{ Wash with dettol/savlon } & \multicolumn{8}{|c|}{ Blocks } & \multirow[t]{2}{*}{ Pooled } \\
\hline & Budgam & Beerwah & Chadoora & Khansahib & Khag & B K Pora & Narbal & Nagam & \\
\hline $\mathbf{n}$ & 40 & 40 & 40 & 40 & 40 & 40 & 40 & 40 & 320 \\
\hline No & $\begin{array}{c}15 \\
(37.50)\end{array}$ & $\begin{array}{c}10 \\
(25.00)\end{array}$ & $\begin{array}{c}13 \\
(32.50)\end{array}$ & $\begin{array}{c}18 \\
(45.00)\end{array}$ & $\begin{array}{c}20 \\
(50.00)\end{array}$ & $\begin{array}{c}19 \\
(47.50)\end{array}$ & $\begin{array}{c}15 \\
(37.50)\end{array}$ & $\begin{array}{c}13 \\
(32.50)\end{array}$ & $\begin{array}{c}123 \\
(38.44)\end{array}$ \\
\hline Yes & $\begin{array}{c}25 \\
(62.50)\end{array}$ & $\begin{array}{c}30 \\
(75.00)\end{array}$ & $\begin{array}{c}27 \\
(67.50)\end{array}$ & $\begin{array}{c}22 \\
(55.00)\end{array}$ & $\begin{array}{c}20 \\
(50.00)\end{array}$ & $\begin{array}{c}21 \\
(52.50)\end{array}$ & $\begin{array}{c}25 \\
(62.50)\end{array}$ & $\begin{array}{c}27 \\
(67.50)\end{array}$ & $\begin{array}{c}197 \\
(61.56)\end{array}$ \\
\hline
\end{tabular}

(Figures in parenthesis indicate percentage, $\mathrm{n}$ indicate sample size $)(\mathrm{P}$ value $=0.279)$

Table.8 Post dog bite Vaccination

\begin{tabular}{|c|c|c|c|c|c|c|c|c|c|}
\hline \multirow[t]{2}{*}{ Post dog bite Vaccination } & \multicolumn{8}{|c|}{ Blocks } & \multirow[t]{2}{*}{ Pooled } \\
\hline & Budgam & Beerwah & Chadoora & Khansahib & Khag & B K Pora & Narbal & Nagam & \\
\hline $\mathbf{n}$ & 40 & 40 & 40 & 40 & 40 & 40 & 40 & 40 & 320 \\
\hline Agree & $\begin{array}{c}4 \\
(10.00)\end{array}$ & $\begin{array}{c}4 \\
(10.00)\end{array}$ & $\begin{array}{c}3 \\
(7.50)\end{array}$ & $\begin{array}{c}4 \\
(10.00)\end{array}$ & $\begin{array}{c}3 \\
(7.50)\end{array}$ & $\begin{array}{c}4 \\
(10.00)\end{array}$ & $\begin{array}{c}4 \\
(10.00)\end{array}$ & $\begin{array}{c}5 \\
(12.50)\end{array}$ & $\begin{array}{c}31 \\
(9.69)\end{array}$ \\
\hline Disagree & $\begin{array}{c}36 \\
(90.00)\end{array}$ & $\begin{array}{c}36 \\
(90.00)\end{array}$ & $\begin{array}{c}37 \\
(92.50)\end{array}$ & $\begin{array}{c}36 \\
(90.00)\end{array}$ & $\begin{array}{c}37 \\
(92.50)\end{array}$ & $\begin{array}{c}36 \\
(90.00)\end{array}$ & $\begin{array}{c}36 \\
(90.00)\end{array}$ & $\begin{array}{c}35 \\
(87.50)\end{array}$ & $\begin{array}{c}289 \\
(90.31)\end{array}$ \\
\hline
\end{tabular}

(Figures in parenthesis indicate percentage, $\mathrm{n}$ indicate sample size $)(\mathrm{P}$ value $=0.997)$

Awareness regarding Brucellosis

Table.9 Knowledge about abortion due to Brucellosis

\begin{tabular}{|c|c|c|c|c|c|c|c|c|c|}
\hline \multirow[t]{2}{*}{ Abortion due to brucellosis in gest. trimester } & \multicolumn{8}{|c|}{ Blocks } & \multirow[t]{2}{*}{ Pooled } \\
\hline & Budgam & Beerwah & Chadoora & Khansahib & Khag & B K Pora & Narbal & Nagam & \\
\hline $\mathbf{n}$ & 40 & 40 & 40 & 40 & 40 & 40 & 40 & 40 & 320 \\
\hline First & $\begin{array}{c}18 \\
(45.00)\end{array}$ & $\begin{array}{c}19 \\
(47.50)\end{array}$ & $\begin{array}{c}22 \\
(55.00)\end{array}$ & $\begin{array}{c}22 \\
(55.00)\end{array}$ & $\begin{array}{c}17 \\
(42.50)\end{array}$ & $\begin{array}{c}17 \\
(42.50)\end{array}$ & $\begin{array}{c}17 \\
(42.50)\end{array}$ & $\begin{array}{c}17 \\
(42.50)\end{array}$ & $\begin{array}{c}149 \\
(46.56)\end{array}$ \\
\hline Last & $\begin{array}{c}22 \\
(55.00)\end{array}$ & $\begin{array}{c}21 \\
(52.50)\end{array}$ & $\begin{array}{c}18 \\
(45.00)\end{array}$ & $\begin{array}{c}18 \\
(45.00)\end{array}$ & $\begin{array}{c}23 \\
(57.50)\end{array}$ & $\begin{array}{c}23 \\
(57.50)\end{array}$ & $\begin{array}{c}23 \\
(57.50)\end{array}$ & $\begin{array}{c}23 \\
(57.50)\end{array}$ & $\begin{array}{c}171 \\
(53.44)\end{array}$ \\
\hline
\end{tabular}

(Figures in parenthesis indicate percentage, $\mathrm{n}$ indicate sample size) $(\mathrm{P}$ value $=0.845)$ 
Table.10 Availability of vaccination against Brucellosis

\begin{tabular}{|c|c|c|c|c|c|c|c|c|c|}
\hline \multirow[t]{2}{*}{ Availability of Vaccine for brucellosis } & \multicolumn{8}{|c|}{ Blocks } & \multirow[t]{2}{*}{ Pooled } \\
\hline & Budgam & Beerwah & Chadoora & Khansahib & Khag & B K Pora & Narbal & Nagam & \\
\hline $\mathbf{n}$ & 40 & 40 & 40 & 40 & 40 & 40 & 40 & 40 & 320 \\
\hline No & $\begin{array}{c}12 \\
(30.00)\end{array}$ & $\begin{array}{c}12 \\
(30.00)\end{array}$ & $\begin{array}{c}11 \\
(27.50)\end{array}$ & $\begin{array}{c}12 \\
(30.00)\end{array}$ & $\begin{array}{c}12 \\
(30.00)\end{array}$ & $\begin{array}{c}16 \\
(40.00)\end{array}$ & $\begin{array}{c}16 \\
(40.00)\end{array}$ & $\begin{array}{c}10 \\
(25.00)\end{array}$ & $\begin{array}{c}101 \\
(31.56)\end{array}$ \\
\hline Yes & $\begin{array}{c}28 \\
(70.00)\end{array}$ & $\begin{array}{c}28 \\
(70.00)\end{array}$ & $\begin{array}{c}29 \\
(72.50)\end{array}$ & $\begin{array}{c}28 \\
(70.00)\end{array}$ & $\begin{array}{c}28 \\
(70.00)\end{array}$ & $\begin{array}{c}24 \\
(60.00)\end{array}$ & $\begin{array}{c}24 \\
(60.00)\end{array}$ & $\begin{array}{c}30 \\
(75.00)\end{array}$ & $\begin{array}{c}219 \\
(68.44)\end{array}$ \\
\hline
\end{tabular}

(Figures in parenthesis indicate percentage, $\mathrm{n}$ indicate sample size $)(\mathrm{P}$ value $=0.789)$

Table.11 Transmission of zoonotic diseases via vegetables and fruits

\begin{tabular}{|c|c|c|c|c|c|c|c|c|c|}
\hline \multirow[t]{2}{*}{ Transmission via veg/fruits } & \multicolumn{8}{|c|}{ Blocks } & \multirow[t]{2}{*}{ Pooled } \\
\hline & Budgam & Beerwah & Chadoora & Khansahib & Khag & B K Pora & Narbal & Nagam & \\
\hline $\mathbf{N}$ & 40 & 40 & 40 & 40 & 40 & 40 & 40 & 40 & 320 \\
\hline Disagree & $\begin{array}{c}34 \\
(85.00)\end{array}$ & $\begin{array}{c}35 \\
(87.50)\end{array}$ & $\begin{array}{c}39 \\
(97.50)\end{array}$ & $\begin{array}{c}39 \\
(97.50)\end{array}$ & $\begin{array}{c}39 \\
(97.50)\end{array}$ & $\begin{array}{c}37 \\
(92.50)\end{array}$ & $\begin{array}{c}38 \\
(95.00)\end{array}$ & $\begin{array}{c}36 \\
(90.00)\end{array}$ & $\begin{array}{c}297 \\
(92.81)\end{array}$ \\
\hline Agree & $\begin{array}{c}6 \\
(15.00)\end{array}$ & $\begin{array}{c}5 \\
(12.50)\end{array}$ & $\begin{array}{c}1 \\
(2.50)\end{array}$ & $\begin{array}{c}1 \\
(2.50)\end{array}$ & $\begin{array}{c}1 \\
(2.50)\end{array}$ & $\begin{array}{c}3 \\
(7.50)\end{array}$ & $\begin{array}{c}2 \\
(5.00)\end{array}$ & $\begin{array}{c}4 \\
(10.00)\end{array}$ & $\begin{array}{c}23 \\
(7.19)\end{array}$ \\
\hline
\end{tabular}

(Figures in parenthesis indicate percentage, $\mathrm{n}$ indicate sample size $)(\mathrm{P}$ value $=0.184)$

Table.12 Exposure of livestock owners to mass media sources (T.V \& Radio) with regard to awareness about zoonotic diseases)

\begin{tabular}{|c|c|c|c|c|c|c|c|c|c|}
\hline \multirow[t]{2}{*}{ Awareness by mass media sources } & \multicolumn{8}{|c|}{ Blocks } & \multirow[t]{2}{*}{ Pooled } \\
\hline & Budgam & Beerwah & Chadoora & Khansahib & Khag & B K Pora & Narbal & Nagam & \\
\hline $\mathbf{n}$ & 40 & 40 & 40 & 40 & 40 & 40 & 40 & 40 & 320 \\
\hline No & $\begin{array}{c}33 \\
(82.50)\end{array}$ & $\begin{array}{c}28 \\
(70.00)\end{array}$ & $\begin{array}{c}31 \\
(77.50)\end{array}$ & $\begin{array}{c}23 \\
(57.50)\end{array}$ & $\begin{array}{c}30 \\
(75.00)\end{array}$ & $\begin{array}{c}27 \\
(67.50)\end{array}$ & $\begin{array}{c}25 \\
(62.50)\end{array}$ & $\begin{array}{c}26 \\
(65.00)\end{array}$ & $\begin{array}{c}223 \\
(69.69)\end{array}$ \\
\hline Yes & $\begin{array}{c}7 \\
(17.50)\end{array}$ & $\begin{array}{c}12 \\
(30.00)\end{array}$ & $\begin{array}{c}9 \\
(22.50)\end{array}$ & $\begin{array}{c}17 \\
(42.50)\end{array}$ & $\begin{array}{c}10 \\
(25.00)\end{array}$ & $\begin{array}{c}13 \\
(32.50)\end{array}$ & $\begin{array}{c}15 \\
(37.50)\end{array}$ & $\begin{array}{c}14 \\
(35.00)\end{array}$ & $\begin{array}{c}97 \\
(30.31)\end{array}$ \\
\hline
\end{tabular}

(Figures in parenthesis indicate percentage, $\mathrm{n}$ indicate sample size) $(\mathrm{P}$ value $=0.246)$ 
Veterinary service seeking

Table.13 Herd testing for zoonotic diseases

\begin{tabular}{|c|c|c|c|c|c|c|c|c|c|}
\hline \multirow[t]{2}{*}{ Herd testing for zoonotic diseases } & \multicolumn{8}{|c|}{ Blocks } & \multirow[t]{2}{*}{ Pooled } \\
\hline & Budgam & Beerwah & Chadoora & Khansahib & Khag & B K Pora & Narbal & Nagam & \\
\hline No & $\begin{array}{c}22 \\
(55.00)\end{array}$ & $\begin{array}{c}22 \\
(55.00)\end{array}$ & $\begin{array}{c}21 \\
(52.50)\end{array}$ & $\begin{array}{c}21 \\
(52.50)\end{array}$ & $\begin{array}{c}24 \\
(60.00)\end{array}$ & $\begin{array}{c}22 \\
(55.00)\end{array}$ & $\begin{array}{c}19 \\
(47.50)\end{array}$ & $\begin{array}{c}19 \\
(47.50)\end{array}$ & $\begin{array}{c}170 \\
(53.13)\end{array}$ \\
\hline Yes & $\begin{array}{c}18 \\
(45.00)\end{array}$ & $\begin{array}{c}18 \\
(45.00)\end{array}$ & $\begin{array}{c}19 \\
(47.50)\end{array}$ & $\begin{array}{c}19 \\
(47.50)\end{array}$ & $\begin{array}{c}16 \\
(40.00)\end{array}$ & $\begin{array}{c}18 \\
(45.00)\end{array}$ & $\begin{array}{c}21 \\
(52.50)\end{array}$ & $\begin{array}{c}21 \\
(52.50)\end{array}$ & $\begin{array}{c}150 \\
(46.88)\end{array}$ \\
\hline
\end{tabular}

(Figures in parenthesis indicate percentage, $\mathrm{n}$ indicate sample size $)(\mathrm{P}$ value $=0.962)$

Table.14 Herd vaccinated for any zoonotic diseases

\begin{tabular}{|c|c|c|c|c|c|c|c|c|c|}
\hline \multirow[t]{2}{*}{ Herd vaccinated for zoonotic disease } & \multicolumn{8}{|c|}{ Blocks } & \multirow[t]{2}{*}{ Poolec } \\
\hline & Budgam & Beerwah & Chadoora & Khansahib & Khag & B K Pora & Narbal & Nagam & \\
\hline $\mathbf{n}$ & 40 & 40 & 40 & 40 & 40 & 40 & 40 & 40 & 320 \\
\hline No & $\begin{array}{c}4 \\
(10.00)\end{array}$ & $\begin{array}{c}2 \\
(5.00)\end{array}$ & $\begin{array}{c}5 \\
(12.50)\end{array}$ & $\begin{array}{c}15 \\
(37.50)\end{array}$ & $\begin{array}{c}5 \\
(12.50)\end{array}$ & $\begin{array}{c}1 \\
(2.50)\end{array}$ & $\begin{array}{c}8 \\
(20.00)\end{array}$ & $\begin{array}{c}6 \\
(15.00)\end{array}$ & $\begin{array}{c}46 \\
(14.38)\end{array}$ \\
\hline Yes & $\begin{array}{c}36 \\
(90.00)\end{array}$ & $\begin{array}{c}38 \\
(95.00)\end{array}$ & $\begin{array}{c}35 \\
(87.50)\end{array}$ & $\begin{array}{c}25 \\
(62.50)\end{array}$ & $\begin{array}{c}35 \\
(87.50)\end{array}$ & $\begin{array}{c}39 \\
(97.50)\end{array}$ & $\begin{array}{c}32 \\
(80.00)\end{array}$ & $\begin{array}{c}34 \\
(85.00)\end{array}$ & $\begin{array}{c}274 \\
(85.63\end{array}$ \\
\hline
\end{tabular}

(Figures in parenthesis indicate percentage, $\mathrm{n}$ indicate sample size $)(\mathrm{P}$ value $=0.001)$

Checklist of livestock rearing safety practices

Table.15 Use of gloves/mask

\begin{tabular}{|c|c|c|c|c|c|c|c|c|c|}
\hline \multirow[t]{2}{*}{ Use of gloves/mask } & \multicolumn{8}{|c|}{ Blocks } & \multirow[t]{2}{*}{ Pooled } \\
\hline & Budgam & Beerwah & Chadoora & Khansahib & Khag & B K Pora & Narbal & Nagam & \\
\hline $\mathbf{n}$ & 40 & 40 & 40 & 40 & 40 & 40 & 40 & 40 & 320 \\
\hline No & $\begin{array}{c}38 \\
(95.00)\end{array}$ & $\begin{array}{c}36 \\
(90.00)\end{array}$ & $\begin{array}{c}37 \\
(92.50)\end{array}$ & $\begin{array}{c}37 \\
(92.50)\end{array}$ & $\begin{array}{c}33 \\
(82.50)\end{array}$ & $\begin{array}{c}26 \\
(65.00)\end{array}$ & $\begin{array}{c}36 \\
(90.00)\end{array}$ & $\begin{array}{c}28 \\
(70.00)\end{array}$ & $\begin{array}{c}271 \\
(84.69)\end{array}$ \\
\hline Yes & $\begin{array}{c}2 \\
(5.00)\end{array}$ & $\begin{array}{c}4 \\
(10.00)\end{array}$ & $\begin{array}{c}3 \\
(7.50)\end{array}$ & $\begin{array}{c}3 \\
(7.50)\end{array}$ & $\begin{array}{c}7 \\
(17.50)\end{array}$ & $\begin{array}{c}14 \\
(35.00)\end{array}$ & $\begin{array}{c}4 \\
(10.00)\end{array}$ & $\begin{array}{c}12 \\
(30.00)\end{array}$ & $\begin{array}{c}49 \\
(15.31)\end{array}$ \\
\hline
\end{tabular}

(Figures in parenthesis indicate percentage, $\mathrm{n}$ indicate sample size $)(\mathrm{P}$ value $=0.001)$ 
Table.16 Proper boiling of milk

\begin{tabular}{|c|c|c|c|c|c|c|c|c|c|}
\hline \multirow[t]{2}{*}{ Proper boiling of milk } & \multicolumn{8}{|c|}{ Blocks } & \multirow[t]{2}{*}{ Pooled } \\
\hline & Budgam & Beerwah & Chadoora & Khansahib & Khag & B K Pora & Narbal & Nagam & \\
\hline $\mathbf{N}$ & 40 & 40 & 40 & 40 & 40 & 40 & 40 & 40 & 320 \\
\hline No & $\begin{array}{c}10 \\
(25.00)\end{array}$ & $\begin{array}{c}6 \\
(15.00)\end{array}$ & $\begin{array}{c}5 \\
(12.50)\end{array}$ & $\begin{array}{c}6 \\
(15.00)\end{array}$ & $\begin{array}{c}6 \\
(15.00)\end{array}$ & $\begin{array}{c}1 \\
(2.50)\end{array}$ & $\begin{array}{c}5 \\
(12.50)\end{array}$ & $\begin{array}{c}2 \\
(5.00)\end{array}$ & $\begin{array}{c}41 \\
(12.81)\end{array}$ \\
\hline Yes & $\begin{array}{c}30 \\
(75.00)\end{array}$ & $\begin{array}{c}34 \\
(85.00)\end{array}$ & $\begin{array}{c}35 \\
(87.50)\end{array}$ & $\begin{array}{c}34 \\
(85.00)\end{array}$ & $\begin{array}{c}34 \\
(85.00)\end{array}$ & $\begin{array}{c}39 \\
(97.50)\end{array}$ & $\begin{array}{c}35 \\
(87.50)\end{array}$ & $\begin{array}{c}38 \\
(95.00)\end{array}$ & $\begin{array}{c}279 \\
(87.19)\end{array}$ \\
\hline
\end{tabular}

(Figures in parenthesis indicate percentage, $\mathrm{n}$ indicate sample size) $(\mathrm{P}$ value $=0.106)$

Table.17 Proper washing of hands after work with animals before eating and before food preparation

\begin{tabular}{|c|c|c|c|c|c|c|c|c|c|}
\hline \multirow[t]{2}{*}{ Proper washing of hands } & \multicolumn{8}{|c|}{ Blocks } & \multirow[t]{2}{*}{ Pooled } \\
\hline & Budgam & Beerwah & Chadoora & Khansahib & Khag & B K Pora & Narbal & Nagam & \\
\hline $\mathbf{N}$ & 40 & 40 & 40 & 40 & 40 & 40 & 40 & 40 & 320 \\
\hline No & $\begin{array}{c}10 \\
(25.00)\end{array}$ & $\begin{array}{c}4 \\
(10.00)\end{array}$ & $\begin{array}{c}12 \\
(30.00)\end{array}$ & $\begin{array}{c}13 \\
(32.50)\end{array}$ & $\begin{array}{c}6 \\
(15.00)\end{array}$ & $\begin{array}{c}9 \\
(22.50)\end{array}$ & $\begin{array}{c}15 \\
(37.50)\end{array}$ & $\begin{array}{c}15 \\
(37.50)\end{array}$ & $\begin{array}{c}84 \\
(26.25)\end{array}$ \\
\hline Yes & $\begin{array}{c}30 \\
(75.00)\end{array}$ & $\begin{array}{c}36 \\
(90.00)\end{array}$ & $\begin{array}{c}28 \\
(70.00)\end{array}$ & $\begin{array}{c}27 \\
(67.50)\end{array}$ & $\begin{array}{c}34 \\
(85.00)\end{array}$ & $\begin{array}{c}31 \\
(77.50)\end{array}$ & $\begin{array}{c}25 \\
(62.50)\end{array}$ & $\begin{array}{c}25 \\
(62.50)\end{array}$ & $\begin{array}{c}236 \\
(73.75)\end{array}$ \\
\hline
\end{tabular}

(Figures in parenthesis indicate percentage, $\mathrm{n}$ indicate sample size) $(\mathrm{P}$ value $=0.040)$

Table.18 Quarantine of animals

\begin{tabular}{|c|c|c|c|c|c|c|c|c|c|}
\hline \multirow[t]{2}{*}{ Quarantine } & \multicolumn{8}{|c|}{ Blocks } & \multirow[t]{2}{*}{ Pooled } \\
\hline & Budgam & Beerwah & Chadoora & Khansahib & Khag & B K Pora & Narbal & Nagam & \\
\hline $\mathbf{n}$ & 40 & 40 & 40 & 40 & 40 & 40 & 40 & 40 & 320 \\
\hline No & $\begin{array}{c}38 \\
(95.00)\end{array}$ & $\begin{array}{c}40 \\
(100.00)\end{array}$ & $\begin{array}{c}40 \\
(100.00)\end{array}$ & $\begin{array}{c}34 \\
(85.00)\end{array}$ & $\begin{array}{c}37 \\
(92.50)\end{array}$ & $\begin{array}{c}38 \\
(95.00)\end{array}$ & $\begin{array}{c}39 \\
(97.50)\end{array}$ & $\begin{array}{c}36 \\
(90.00)\end{array}$ & $\begin{array}{c}302 \\
(94.38)\end{array}$ \\
\hline Yes & $\begin{array}{c}2 \\
(5.00)\end{array}$ & $\begin{array}{c}0 \\
(0.00)\end{array}$ & $\begin{array}{c}0 \\
(0.00)\end{array}$ & $\begin{array}{c}6 \\
(15.00)\end{array}$ & $\begin{array}{c}3 \\
(7.50)\end{array}$ & $\begin{array}{c}2 \\
(5.00)\end{array}$ & $\begin{array}{c}1 \\
(2.50)\end{array}$ & $\begin{array}{c}4 \\
(10.00)\end{array}$ & $\begin{array}{c}18 \\
(5.63)\end{array}$ \\
\hline
\end{tabular}

(Figures in parenthesis indicate percentage, $\mathrm{n}$ indicate sample size $)(\mathrm{P}$ value $=0.053)$ 
Table.19 Preventive measures with regard to sick, weak, pregnant and old

\begin{tabular}{|c|c|c|c|c|c|c|c|c|c|}
\hline Avoid working with animals & \multicolumn{9}{|c|}{ Blocks } \\
\hline & Budgam & Beerwah & Chadoora & Khansahib & Khag & B K Pora & Narbal & Nagam & Pooled \\
\hline n & $\mathbf{4 0}$ & $\mathbf{4 0}$ & $\mathbf{4 0}$ & $\mathbf{4 0}$ & $\mathbf{4 0}$ & $\mathbf{4 0}$ & $\mathbf{4 0}$ & $\mathbf{4 0}$ & $\mathbf{3 2 0}$ \\
\hline No & 28 & 25 & 23 & 18 & 24 & 28 & 29 & 27 & 202 \\
& $(70.00)$ & $(62.50)$ & $(57.50)$ & $(45.00)$ & $(60.00)$ & $(70.00)$ & $(72.50)$ & $(67.50)$ & $(63.13)$ \\
\hline Yes & 12 & 15 & 17 & 22 & 16 & 12 & 11 & 13 & 118 \\
& $(30.00)$ & $(37.50)$ & $(42.50)$ & $(55.00)$ & $(40.00)$ & $(30.00)$ & $(27.50)$ & $(32.50)$ & $(36.88)$ \\
\hline
\end{tabular}

(Figures in parenthesis indicate percentage, $\mathrm{n}$ indicate sample size) $(\mathrm{P}$ value $=0.198)$

Table.20 Animal shelter housing

\begin{tabular}{|c|c|c|c|c|c|c|c|c|c|}
\hline Animal shelter housing & \multicolumn{9}{|c|}{ Blocks } \\
\hline & Budgam & Beerwah & Chadoora & Khansahib & Khag & B K Pora & Narbal & Nagam \\
\hline $\mathbf{n}$ & $\mathbf{4 0}$ & $\mathbf{4 0}$ & $\mathbf{4 0}$ & $\mathbf{4 0}$ & $\mathbf{4 0}$ & $\mathbf{4 0}$ & $\mathbf{4 0}$ & $\mathbf{4 0}$ & $\mathbf{3 2 0}$ \\
\hline No & 7 & 1 & 1 & 1 & 0 & 2 & 4 & 4 & 20 \\
& $(17.50)$ & $(2.50)$ & $(2.50)$ & $(2.50)$ & $(0.00)$ & $(5.00)$ & $(10.00)$ & $(10.00)$ & $(6.25)$ \\
\hline Yes & 33 & 39 & 39 & 39 & 40 & 38 & 36 & 36 & 300 \\
& $(82.50)$ & $(97.50)$ & $(97.50)$ & $(97.50)$ & $(100.00)$ & $(95.00)$ & $(90.00)$ & $(90.00)$ & $(93.75)$ \\
\hline
\end{tabular}

(Figures in parenthesis indicate percentage, $\mathrm{n}$ indicate sample size) $(\mathrm{P}$ value $=0.023$ )

Table.21 Animal segregation

\begin{tabular}{|c|c|c|c|c|c|c|c|c|c|}
\hline Animal segregation & \multicolumn{9}{|c|}{ Blocks } \\
\hline & Budgam & Beerwah & Chadoora & Khansahib & Khag & B K Pora & Narbal & Nagam \\
\hline $\mathbf{n}$ & $\mathbf{4 0}$ & $\mathbf{4 0}$ & $\mathbf{4 0}$ & $\mathbf{4 0}$ & $\mathbf{4 0}$ & $\mathbf{4 0}$ & $\mathbf{4 0}$ & $\mathbf{4 0}$ & $\mathbf{3 2 0}$ \\
\hline No & 33 & 16 & 18 & 7 & 8 & 9 & 13 & 16 & 120 \\
& $(82.50)$ & $(40.00)$ & $(45.00)$ & $(17.50)$ & $(20.00)$ & $(22.50)$ & $(32.50)$ & $(40.00)$ & $(37.50)$ \\
\hline Yes & 7 & 24 & 22 & 33 & 32 & 31 & 27 & 24 & 200 \\
& $(17.50)$ & $(60.00)$ & $(55.00)$ & $(82.50)$ & $(80.00)$ & $(77.50)$ & $(67.50)$ & $(60.00)$ & $(62.50)$ \\
\hline
\end{tabular}

(Figures in parenthesis indicate percentage, $\mathrm{n}$ indicate sample size) $(\mathrm{P}$ value $=0.001)$ 
Table.22 Public health sanitation

\begin{tabular}{|c|c|c|c|c|c|c|c|c|c|}
\hline Public health sanitation & \multicolumn{9}{|c|}{ Blocks } \\
\hline & Budgam & Beerwah & Chadoora & Khansahib & Khag & B K Pora & Narbal & Nagam \\
\hline $\mathbf{n}$ & $\mathbf{4 0}$ & $\mathbf{4 0}$ & $\mathbf{4 0}$ & $\mathbf{4 0}$ & $\mathbf{4 0}$ & $\mathbf{4 0}$ & $\mathbf{4 0}$ & $\mathbf{4 0}$ & $\mathbf{3 2 0}$ \\
\hline No & 22 & 29 & 26 & 27 & 25 & 22 & 25 & 31 & 207 \\
& $(55.00)$ & $(72.50)$ & $(65.00)$ & $(67.50)$ & $(62.50)$ & $(55.00)$ & $(62.50)$ & $(77.50)$ & $(64.69)$ \\
\hline Yes & 18 & 11 & 14 & 13 & 15 & 18 & 15 & 9 & 113 \\
& $(45.00)$ & $(27.50)$ & $(35.00)$ & $(32.50)$ & $(37.50)$ & $(45.00)$ & $(37.50)$ & $(22.50)$ & $(35.31)$ \\
\hline
\end{tabular}

(Figures in parenthesis indicate percentage, $\mathrm{n}$ indicate sample size $)(\mathrm{P}$ value $=0.375)$

Table.23 Disposal of diseased/dead animals

\begin{tabular}{|c|c|c|c|c|c|c|c|c|c|}
\hline \multirow[t]{2}{*}{ Disposal of diseased/dead animals } & \multicolumn{8}{|c|}{ Blocks } & \multirow[t]{2}{*}{ Pooled } \\
\hline & Budgam & Beerwah & Chadoora & Khansahib & Khag & B K Pora & Narbal & Nagam & \\
\hline $\mathbf{N}$ & 40 & 40 & 40 & 40 & 40 & 40 & 40 & 40 & 320 \\
\hline No & $\begin{array}{c}15 \\
(37.50)\end{array}$ & $\begin{array}{c}14 \\
(35.00)\end{array}$ & $\begin{array}{c}16 \\
(40.00)\end{array}$ & $\begin{array}{c}16 \\
(40.00)\end{array}$ & $\begin{array}{c}13 \\
(32.50)\end{array}$ & $\begin{array}{c}20 \\
(50.00)\end{array}$ & $\begin{array}{c}19 \\
(47.50)\end{array}$ & $\begin{array}{c}11 \\
(27.50)\end{array}$ & $\begin{array}{c}124 \\
(38.75)\end{array}$ \\
\hline Yes & $\begin{array}{c}25 \\
(62.50)\end{array}$ & $\begin{array}{c}26 \\
(65.00)\end{array}$ & $\begin{array}{c}24 \\
(60.00)\end{array}$ & $\begin{array}{c}24 \\
(60.00)\end{array}$ & $\begin{array}{c}27 \\
(67.50)\end{array}$ & $\begin{array}{c}20 \\
(50.00)\end{array}$ & $\begin{array}{c}21 \\
(52.50)\end{array}$ & $\begin{array}{c}29 \\
(72.50)\end{array}$ & $\begin{array}{c}196 \\
(61.25)\end{array}$ \\
\hline
\end{tabular}

(Figures in parenthesis indicate percentage, $\mathrm{n}$ indicate sample size) $(\mathrm{P}$ value $=0.479)$

Table.24 Anti-rabies vaccination of farmers

\begin{tabular}{|c|c|c|c|c|c|c|c|c|c|}
\hline Anti-rabies vaccine & \multicolumn{9}{|c|}{ Blocks } \\
\hline & Budgam & Beerwah & Chadoora & Khansahib & Khag & B K Pora & Narbal & Nagam \\
\hline n & $\mathbf{4 0}$ & $\mathbf{4 0}$ & $\mathbf{4 0}$ & $\mathbf{4 0}$ & $\mathbf{4 0}$ & $\mathbf{4 0}$ & $\mathbf{4 0}$ & $\mathbf{4 0}$ & $\mathbf{3 2 0}$ \\
\hline No & 8 & 5 & 2 & 2 & 13 & 7 & 21 & 12 & 70 \\
& $(20.00)$ & $(12.50)$ & $(5.00)$ & $(5.00)$ & $(32.50)$ & $(17.50)$ & $(52.50)$ & $(30.00)$ & $(21.88)$ \\
\hline Yes & 32 & 35 & 38 & 38 & 27 & 33 & 19 & 28 & 250 \\
& $(80.00)$ & $(87.50)$ & $(95.00)$ & $(95.00)$ & $(67.50)$ & $(82.50)$ & $(47.50)$ & $(70.00)$ & $(78.13)$ \\
\hline
\end{tabular}

(Figures in parenthesis indicate percentage, $\mathrm{n}$ indicate sample size) $(\mathrm{P}$ value $=0.001$ ) 
Table.25 Cleaning and disinfection of animal sheds

\begin{tabular}{|c|c|c|c|c|c|c|c|c|c|}
\hline \multirow[t]{2}{*}{ Cleaning and disinfection of animal sheds } & \multicolumn{8}{|c|}{ Blocks } & \multirow[t]{2}{*}{ Pooled } \\
\hline & Budgam & Beerwah & Chadoora & Khansahib & Khag & B K Pora & Narbal & Nagam & \\
\hline $\mathbf{N}$ & 40 & 40 & 40 & 40 & 40 & 40 & 40 & 40 & 320 \\
\hline No & $\begin{array}{c}1 \\
(2.50)\end{array}$ & $\begin{array}{c}0 \\
(0.00)\end{array}$ & $\begin{array}{c}1 \\
(2.50)\end{array}$ & $\begin{array}{c}2 \\
(5.00)\end{array}$ & $\begin{array}{c}4 \\
(10.00)\end{array}$ & $\begin{array}{c}1 \\
(2.50)\end{array}$ & $\begin{array}{c}0 \\
(0.00)\end{array}$ & $\begin{array}{c}0 \\
(0.00)\end{array}$ & $\begin{array}{c}9 \\
(2.81)\end{array}$ \\
\hline Yes & $\begin{array}{c}39 \\
(97.50)\end{array}$ & $\begin{array}{c}40 \\
(100.00)\end{array}$ & $\begin{array}{c}39 \\
(97.50)\end{array}$ & $\begin{array}{c}38 \\
(95.00)\end{array}$ & $\begin{array}{c}36 \\
(90.00)\end{array}$ & $\begin{array}{c}39 \\
(97.50)\end{array}$ & $\begin{array}{c}40 \\
(100.00)\end{array}$ & $\begin{array}{c}40 \\
(100.00)\end{array}$ & $\begin{array}{c}311 \\
(97.19)\end{array}$ \\
\hline
\end{tabular}

(Figures in parenthesis indicate percentage, $\mathrm{n}$ indicate sample size) $(\mathrm{P}$ value $=0.108)$

Table.26 Health Hazard through working with animals

\begin{tabular}{|c|c|c|c|c|c|c|c|c|c|}
\hline \multirow{2}{*}{$\begin{array}{l}\text { Health Hazard through working } \\
\text { with animals }\end{array}$} & \multicolumn{8}{|c|}{ Blocks } & \multirow[t]{2}{*}{ Pooled } \\
\hline & Budgam & Beerwah & $\begin{array}{c}\text { Chado } \\
\text { ora }\end{array}$ & $\begin{array}{c}\text { Khansahi } \\
\text { b }\end{array}$ & Khag & $\begin{array}{c}\text { B K } \\
\text { Pora }\end{array}$ & Narbal & Nagam & \\
\hline $\mathbf{N}$ & 40 & 40 & 40 & 40 & 40 & 40 & 40 & 40 & 320 \\
\hline No & $\begin{array}{c}38 \\
(95.00)\end{array}$ & $\begin{array}{c}38 \\
(95.00)\end{array}$ & $\begin{array}{c}38 \\
(95.00)\end{array}$ & $\begin{array}{c}39 \\
(97.50)\end{array}$ & $\begin{array}{c}40 \\
(100.00)\end{array}$ & $\begin{array}{c}40 \\
(100.00)\end{array}$ & $\begin{array}{c}38 \\
(95.00)\end{array}$ & $\begin{array}{c}38 \\
(95.00)\end{array}$ & $\begin{array}{c}309 \\
(96.56)\end{array}$ \\
\hline Yes & $\begin{array}{c}2 \\
(5.00)\end{array}$ & $\begin{array}{c}2 \\
(5.00)\end{array}$ & $\begin{array}{c}2 \\
(5.00)\end{array}$ & $\begin{array}{c}1 \\
(2.50)\end{array}$ & $\begin{array}{c}0 \\
(0.00)\end{array}$ & $\begin{array}{c}0 \\
(0.00)\end{array}$ & $\begin{array}{c}2 \\
(5.00)\end{array}$ & $\begin{array}{c}2 \\
(5.00)\end{array}$ & $\begin{array}{c}11 \\
(3.44)\end{array}$ \\
\hline
\end{tabular}

(Figures in parenthesis indicate percentage, $\mathrm{n}$ indicate sample size $)(\mathrm{P}$ value $=0.730)$ 
Comparing the overall knowledge level of livestock farmers of different blocks maximum knowledge level of livestock farmers was observed in Budgam, Beerwah, B.K Pora, Chadoora and Nagam block while as minimum level of knowledge was in Khag and Narbal block. The difference was statistically significant.

From overall study it was revealed that knowledge and awareness regarding transmission, modes of transmission and risks related to zoonotic diseases was found limited at some levels. This stressed on the need for providing better knowledge for effective control of zoonosis.

\section{References}

Adesiji,Y.o., Adesiji, G.B. and Fagbami, A.H. 2005. Knowledge, awareness and practices among occupationally exposed individuals in Osun state. Science Focus, 10(1): 38-41.

Ananthanarayan, R. and Paniker, J. 2013. Brucella. In: Arti, K., editor. Textbook of Microbiology. $9^{\text {th }}$ ed. University press, Hyderabad, 340-343.

Asbjer E. 2009. Dog population management in Malawi and Peri. Projectreport, Department of Biomedical Sciences and Veterinary Public Health. Swedish University of Agricultural Sciences, International Journal of Recent Scientific Research, 6: 5131-5138.

Dean A. S., Crump L., Greter, H., Hattendorf, J., Schelling, E. and Zinsstag, J. 2012. Clinical manifestations of human brucellosis: a systematic review and meta-analysis. PLOS Neglected Tropical Diseases, 6: 1929.

Haagsma, J.A., Tariq, L., Heederik, D.J. and Havelaar, A. H. 2011. Infectious disease risks associated with occupational exposure: A systematic review of the literature. Occupational Environmental Medicine, 6(3):111-115.

International Livestock Research Institute (ILRI). 2012. Mapping of poverty and likely zoonoses hotspots. Zoonoses Project: Report to Department for International Development in Nairobi, Kenya. International Livestock Research Institute, 119.

Junaidu, M. M. Y., Bhagavandas, M., Yusha, K. and Umar, U. 2015. Study of knowledge, attitude and practices regarding hygiene among abattoir workers in Kano state metropolitan, Nigeria.International Journal of Science and Research, 4(1): 2474-2478.

Molyneux, D., Hallaj, Z., Keusch, G. T., Mcmanus, D. P., Ngowi, H., Cleaveland, S. and Ramos-Jimenez. 2011. Zoonoses and marginalised infectious diseases of poverty: where do we stand.Parasites and Vectors, 4: 106.

Nkuchia, M M, Ruth L, Chris A B and Henriette, V. 2007. Infectious diseases surveillance. Blackwell Publishing, 2: 246-248.

Schelling, E., Diguimbaye, C., Daoud, S., Nicolet, J., Boerlin, P., Tanner, M. and Zinsstag, J. 2003.Brucellosis and Qfever Seroprevalances of Nomadic Pastoralists and their Livestock in Chad. Preventive Veterinary Medicine, 61: 279-293.

Smiths H L and Cutler S J. 2004. Contributions of Biotechnology to the control and prevention of brucellosis in Africa . African Journal of Biotechnology, 3: 631-639.

Swai, E. S., Schoonman, L. and Daborn, C. J. 2010.Knowledge and attitude towards zoonoses among animal health workers and livestock keepers in Arusha and Tanga, Tanzania.Journal of Health Research, 12(4): 205-209. 


\section{How to cite this article:}

Insha Mir and Abdul Hai Bhat. 2020. Awareness of Zoonotic Diseases among Livestock Farmers in Budgam District of Kashmir Valley. Int.J.Curr.Microbiol.App.Sci. 9(05): 296-309 . doi: https://doi.org/10.20546/ijcmas.2020.905.032 